\title{
When apathy is deadlier than COVID-19
}

To the Editor - Throughout the COVID-19 pandemic, many have disregarded worry since the virus "only kills old and disabled people" ${ }^{\prime 1}$. Aside from being inaccurate, this reflects the most disturbing theme across COVID-19: society prioritizing comfort and convenience over the safety of vulnerable groups, apparently deemed disposable. The impact of these positions has been seen in long-term care homes (LTCHs) globally ${ }^{2}$ and significantly in $\mathrm{Canada}^{3}$, where LTCH residents disproportionately represent $81 \%$ of Canadian COVID-19 deaths, more than double the average (38\%) of all other Organisation for Economic Cooperation and Development (OECD) countries ${ }^{3}$. Although Canada reports the worst proportion of COVID-19 deaths linked to LTCHs globally, all nations exhibit a concerning trend for the pandemic's trajectory where society's most vulnerable are suffering the consequences of apathy.

Apathetic attitudes towards LTCHs existed long before being exposed by COVID-19 (refs. ${ }^{4,5}$ ). Inadequate LTCH funding from provincial governments and pandemic preparation revealed three existing systemic risk factors for poor outcomes among LTCH residents (Fig. 1) 1). $^{3,6}$. First, LTCHs are structured so residents share rooms (two to four individuals per room $)^{3}$, making physical distancing impossible. Second, the employment model used in LTCHs require personal support workers (PSWs) to be responsible for multiple residents, with at least ten and as many as over 40 residents in their care ${ }^{7}$. Additionally, since PSWs receive low pay and are rarely scheduled on a full-time basis ${ }^{8}$, they must work in several LTCHs to make a living wage, possibly contributing to the spread of COVID-19 between $\mathrm{LTCHs}^{9,10}$. Third, personal protective equipment (PPE) was in short supply during the pandemic's first wave ${ }^{10}$. The Canadian military, deployed to LTCHs in crisis during COVID-19, reported a "culture of fear" ${ }^{\text {"11 }}$ related to using PPE due to the cost of supplies. The doing more with less' model also applies to seniors and persons with disabilities receiving PSW services in the community, leaving them vulnerable to an uncontrolled outbreak.

LTCHs suspended the right of families and caregivers to visit in an attempt to prevent further exposure to COVID-19. Residents lost access to their loved ones, socialization ${ }^{12}$ and, most importantly, their personal advocates, by relegating family caregivers to visit through window

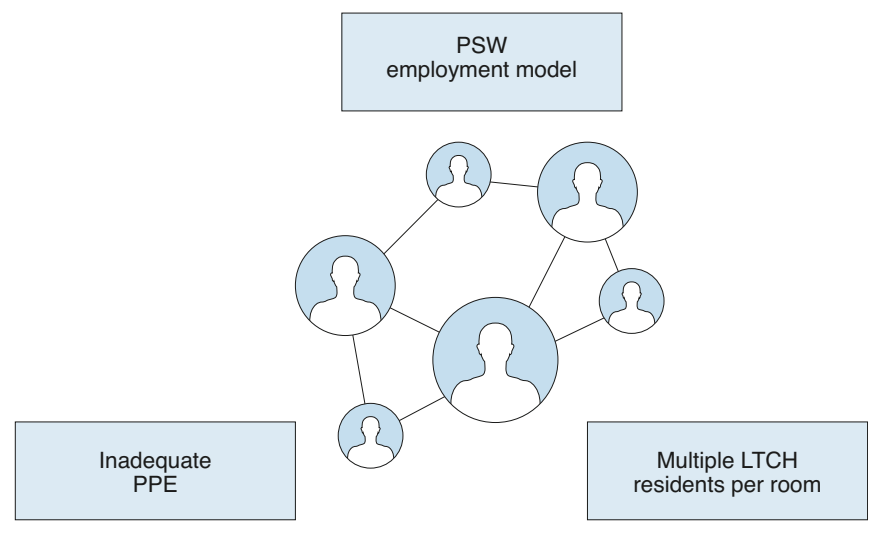

Fig. 1 | Systemic risk factors leading to disproportionate COVID-19 cases and mortality among LTCH residents. Interaction of systematic risk factors, such as inadequate PPE, the PSW employment model and multiple LTCH residents per room, likely contributes to outbreaks of COVID-19 and disproportionate deaths among $\mathrm{LTCH}$ residents.

panes $^{13}$. Without the watchful eye of family and friends, there have been reports of inadequate end-of-life care for residents dying from COVID-19 and non-COVID-19 causes, with some residents being refused treatment in hospitals ${ }^{14}$ or the option to leave their LTCH. While restrictions were temporarily lifted over the summer, they have been reimplemented, with visits only being allowed for one 'essential' caregiver per resident ${ }^{13}$.

Despite the clear warning of an impending pandemic and previous knowledge of the risk factors in LTCHs, governments and LTCH administrations did nothing to change care modalities which could have protected this population. By October, when the anticipated second wave of COVID-19 engulfed the country, we had seen outbreaks in $30 \%$ of Ontario LTCHs $^{15}$. Unsurprisingly, preparations fell short in many jurisdictions ${ }^{16}$, leaving LTCHs understaffed and COVID-19 testing centres overwhelmed. Although the Ontario provincial government committed additional funding ${ }^{17}$ for LTCHs in December 2020 , implementation will take place over four years with little immediate effect while LTCH residents continue to die at appalling rates. We can no longer claim to be 'shocked' by these events when the human life belonging to vulnerable groups is so frequently devalued.

To paraphrase a popular adage, we can learn much about a society by how the most vulnerable are treated. The pandemic has made it clear that we must change how the healthcare system provides support to seniors and people with disabilities. Across the world, systemic factors have been revealed, continuing to place the most vulnerable at unacceptable risk. Petitioning governments should not fall just to those directly affected, such as families of LTCH residents. Local officials have a responsibility to proactively improve the situation for those likely to lose the most, responding to the international calls to action from the World Health Organization and the United Nations ${ }^{18}$. While there is a spark of hope with the prioritization of LTCH residents for vaccine roll-out in Canada ${ }^{19}$, this is dimmed by the recollection that the same government allowed thousands of vulnerable Canadians to die unnecessarily. Lest we forget our parents and grandparents, and remember that they deserve dignity and protection.

\footnotetext{
Catherine Stratton $1,2 \bowtie$, Lene Andersen², Laurie Proulx ${ }^{2,3}$ and Emily Sirotich2,3,4

${ }^{1}$ Department of Chronic Disease Epidemiology, Yale School of Public Health, New Haven, CT, USA.

${ }^{2}$ Disability Equity in Health, Ottawa, Ontario, Canada. ${ }^{3}$ Canadian Arthritis Patient Alliance, Ottawa, Ontario, Canada. ${ }^{4}$ Department of Health Research Methods, Evidence, and Impact, McMaster University Faculty of Health Sciences, Hamilton, Ontario, Canada.

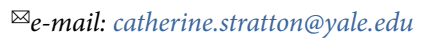


Published online: 11 February 2021

https://doi.org/10.1038/s43587-021-00030-w

References

1. Xiang, X. et al. J. Gerontol. B-Psychol. https://doi.org/10.1093/ geronb/gbaal28 (2020).

2. Grey, S. \& Macaskill, A. In Shielding its Hospitals from COVID-19, Britain Left Many of the Weakest Exposed (Reuters, 2020).

3. Pandemic Experience in the Long-Term Care Sector: How Does Canada Compare With Other Countries? (Canadian Institute for Health Information, 2020).

4. Transforming Long-term Care to Keep Residents Healthy and Safe (Registered Nurses' Association of Ontario, 2018).

5. Hawes, C. In Elder Mistreatment: Abuse, Neglect, and Exploitation in an Aging America (eds Bonnie, R. J. \& Wallace, R. B.) (National Academies Press, 2003).

6. Estabrooks, C. A. et al. Restoring Trust: COVID-19 and the Future of Long-term Care (Royal Society of Canada, 2020).

7. Aitken, J. 5 answers to questions about long-term care homes in Ontario. Retire-At-Home http://bit.ly/2XSrWuh (2017)
8. Labour group calls for premium wage increase for personal support workers. CBC News http://bit.ly/39suN2e (2020).

9. Van Houtven, C. H., DePasquale, N. \& Coe, N. B. J. Am. Geriatr. Soc. 68, 1657-1660 (2020).

10. Fisman, D. N. et al. JAMA Netw. Open 3, e2015957 (2020).

11. Campion-Smith, B., Ferguson, R. \& Benzie, R. Canada's military exposes 'extremely troubling' conditions in Ontario long-term-care homes. Toronto Star http://bit.ly/3oNJ93Q (2020)

12. Armitage, R. \& Nellums, L. B. Lancet Public Health 5, e256 (2020).

13. Davidson, S. Ontario tightens visitor policies at long-term care homes. Here are the new rules CTV News http://bit.ly/3bFxT5S (2020).

14. Payne, E. Only $13 \%$ of Ontario's long-term care COVID patients went to hospital; advocates want to know why. Ottawa Citizen http://bit.ly/3bDhhLZ (2020).

15. COVID-19 cases: long-term care homes. Government of Ontario http://bit.ly/3qonfEm (2020)

16. Dyer, E. The great PPE panic: how the pandemic caught Canada with its stockpiles down. CBC News http://bit.ly/3bAODep (2020)

17. Ferguson, R. Doug Ford commits up to $\$ 1.9$ billion a year to give nursing-home residents more hands-on care. Toronto Star http:// bit.ly/3nEU5iB (2020)
18. Promoting the rights and dignity of older persons during COVID-19 and beyond. United Nations http://bit.ly/3bz8XwK (2020).

19. Lieberman, C. Toronto's 1st COVID-19 vaccination clinic opens for long-term care home staff. Global News http://bit.ly/2LTSZCn (2020).

\section{Author contributions}

C.S., L.A., L.P. and E.S. contributed to the conception of the idea for this commentary. C.S., L.A., L.P. and E.S. contributed to composing the manuscript. C.S. finalized the formatting and submitted the manuscript. C.S., L.A., L.P. and E.S. agree that any questions related to the accuracy or integrity of any part of the work are appropriately investigated and resolved.

Competing interests

The authors declare no competing interests. 